\title{
Biomass-Diversity Responses and Spatial Dependencies in Disturbed Tallgrass Prairies
}

\author{
JaVIER G. P. GAMARRA*† AND RicARd V. SOLÉ $\dagger$ \\ *Complex Systems Research Group - FEN, Universitat Politècnica de Catalunya, Campus Nord \\ B4-B5, 08034, Barcelona, Spain and †Santa Fe Institute, 1399 Hyde Park Road, Santa Fe, \\ NM 87501, U.S.A.
}

(Received on 15 October 2001, Accepted in revised form on 19 December 2001)

\begin{abstract}
Monotonic, hump-shaped and zero-correlation productivity-diversity relationships have been found to date in many ecosystems. This diversity of responses has puzzled ecologists in their search for general principles on ecosystem functioning. Some state that the scale of observation is crucial in defining this relationship. We have developed a spatial model of tallgrass prairies where biomass and litter dynamics are defined by uncoupled difference equations. In this system, we periodically apply prescribed fire as a disturbance that propagates through neighboring cells. The model shows percolation thresholds at points where small-scale spatial heterogeneity and large-scale, global correlation coexist, resulting in power-law distributions in available areas for non-dominant species. These points maximize the biomass-diversity relationship. Our results suggest that spatial dependencies and the disturbance heterogeneity hypothesis are the cornerstone processes accounting for unimodality in productivity-diversity relationships.
\end{abstract}

(C) 2002 Elsevier Science Ltd. All rights reserved.

\section{Introduction}

The diversity-productivity relationship in ecosystems has been a source of continuous debate (Tilman, 1982; Rosenzweig \& Abramsky, 1993; Tilman \& Pacala, 1993; Huston, 1994; Abrams, 1995; Guo \& Berry, 1998). Either hump-shaped (Rosenzweig \& Abramsky, 1993), monotonic (Naeem et al., 1994; Tilman et al., 1996; Hector et al., 1999) or no-relationship (Johnson et al., 1996) have been documented. The consequences of the declining diversity that ecosystems around the world are facing through human action are very different depending on which is the true relationship, if any. For example, yield shortages

$\dagger$ Author to whom correspondence should be addressed. E-mail: javier@complex.upc.es of many ecosystems would be predicted under the monotonic increasing hypothesis. However, hump-shaped curves would require the need for local actions depending on the part of the curve where any community is inserted.

However, sampling effects and specificity of plants or functional types could invalidate some of the previous results (Tilman \& Pacala, 1993). Recently, some studies addressed the importance of scale-dependent properties in this relationship (Waide et al., 1999; Gross et al., 2000). These findings could cast in doubt the robustness of former studies, which have been mostly performed on naturalized grasslands through independent plots. However, their experimental tractability still relies on the high diversity levels that can be accomplished in very small sample 
areas and the high turnover rates experienced in grasslands.

The two most striking features that many grasslands present are a high interannual variability in aboveground net primary production (ANPP) and precipitation (Seastedt \& Knapp, 1993; Knapp \& Smith, 2001). Thus, the tallgrass prairie represents a useful test tube for experimentally proving many relationships involving diversity issues over wide ranges of productivity and precipitation values.

In fact, these characteristics have conveyed ecologists to suspect that high growth rates alone can be a first signal of the existence of strong internal nonlinearities. These drive the system to reach a wide spectrum of yield values over time with no need to account for large variabilities in external disturbances. A lack of convincing correlation between precipitation, soil nitrogen $(\mathrm{N})$, and yield patterns (Briggs \& Knapp, 1995; O'Lear et al., 1996) suggest that maximum growth rates are attained by simultaneous release from more than one limiting resource (the so-called transient maxima hypothesis) (Seastedt \& Knapp, 1993). However, under experimental conditions, Tilman \& Wedin (1991) showed that increasing soil $\mathrm{N}$ availability may correspond with a period-doubling cascade in Agrostis scabra aboveground biomass production and consequently modelled the system as a single difference equation.

Besides external disturbances such as high intra and interannual variability in precipitation patterns (Seastedt \& Knapp, 1993), the presence of large herbivores reducing biomass (Knapp et al., 1999) and the commonness of fire (Axelrod, 1985; Huston, 1994) are also inherent components landscaping these ecosystems. Prescribed fire has been historically used as a tool for livestock and tallgrass management (Daubenmire, 1968; Culver, 1998), used mainly for prairie maintenance. It stabilizes or destabilizes vegetation depending on fuel availability and species composition (Culver, 1998). The first question arising is: how are related external disturbances (such as fire) and strong internal nonlinearities governing an ecosystem? and, how this relationship affects the spatial distribution of biomass and species and the biomass-diversity relationship in that ecosystem?
Spatial dependencies arising in the system may well be of critical importance in answering these questions. First, when space is not considered, nonlinear systems undergoing chaotic motion, if affected by periodic external disturbances, can be controlled, even under the presence of demographic stochasticity. Control depends on certain parameter combinations, mainly frequency and intensity of the disturbance. These chaos control techniques have been scarcely used in the theoretical ecology literature (Solé et al., 1999; Gamarra et al., 2001) and have been once used as platforms for the experimental testing of chaos in ecological systems (Desharnais et al., 2001). Second, by introducing space, an overwhelming range of biomass and diversity patterns could appear as a consequence of spatial correlations. Chaos control has also been successfully applied on spatial domains via a threespecies model in a coupled map lattice by adding periodic disturbances on regularly spaced cells (Gamarra et al., 2001). These disturbances broke up the large, globally correlating chaotic waves that are typical under no control schemes and imposed the domain of local correlations over the system.

Very simple cellular automata models of disturbance have shown that the coupling of local dispersal and fire promotes clumping and the persistence of non-competitive species (Green, 1989, 1990), and maintain high species richness. The use of fire in models has a paradigm in the so-called forest fire model (Bak et al., 1990; Drossel \& Schwabl, 1992). These models predict the formation of large correlations, self-organization towards critical percolation thresholds (i.e. those tree density values where a path of neighboring burned trees crosses the lattice), and the formation of fractal structures over a wide range of scales. Under some assumptions on life-history trade-offs (i.e. competition for available space-dispersal) and recruitment limitation (Tilman, 1994; Hurtt \& Pacala, 1995; Solé \& Alonso, 2000), these processes might result in high diversity levels. Actually, fractal properties have been properly identified in real forests subject to eventual fires (Malamud et al., 1998).

However, none of these models have dealt with the interaction of local, nonlinear motion 
and the correlating effects of external disturbances applied globally in the landscape. Such an interaction is probably the cornerstone process taking place in highly productive systems affected by large variabilities in the external forces affecting the system. These forces include fire (Axelrod, 1985) or grazing (Milchunas et al., 1988), and are very often the leading factors giving rise to largescale spatial correlations, which have been shown to be essential for the increase of $\beta$-diversity while $\alpha$-diversity hardly changed.

The main questions we are addressing in our study are: (1) can the yield of tallgrass patches be enhanced under different soil $\mathrm{N}$ availability conditions by means of periodic, small disturbances on biomass or litter? (2) Assuming that high spatial heterogeneity and spatial dependence (large correlations in space and time) (Henebry, 1995) maximize diversity, are there specific disturbance conditions under which diversity (i.e. high spatial heterogeneity and dependence) and yield can be maximized and stable (Collins, 2000)? (3) How do spatial dependencies affect the biomass-diversity relationship?

In our study, we have focused on the tallgrass prairie as a model ecosystem where local nonlinearities and fire (as a global environmental disturbance) take place. The questions of this study are based on two possible scenarios. One, typical from upland sites where $\mathrm{N}$ availability is scarce and thus, stable motion of biomass and litter governs, and the other, undergoing chaotic motion due to high $\mathrm{N}$ availability, typical from lowland sites (Briggs \& Knapp, 1995). We study a model example where we use prescribed fire as a commonly used management tool by coupling a lattice of independent cells with a cellular automaton formalism for the spread of fire. This spatial model aims to predict the behavior and consequences of prescribed fire as a form of control on productivity and diversity.

\section{A Spatial Model for Prescribed Fire}

The main features distinguishing our model are that it is an interacting particle system where fire can spread over a lattice where the states of the cells can have infinite values. CA models, instead, only have two states (i.e. where cells takes values 1 for burned state or 0 for unburned state) like the Bak et al. (1990) forest fire model. The model described here is a typical CA forest fire model that runs on a system with local, independent dynamics where every cell takes the dynamics described by Tilman \& Wedin (1991) and Bascompte \& Rodriguez (2000):

$$
L_{t+1}(i)=p L_{t}(i)+k B_{t+1}(i),
$$

where $L_{t}(i)$ is litter biomass at year $t$ and $1 \times 1 \mathrm{~m}$ cell position $i, p$ is the finite rate of persistence of litter and $k$ is the rate of biomass-litter conversion. The biomass (heretofore denoted as $B$ ) at year $t$ depends on $L_{t-1}$ such that

$$
B_{t+1}(i)=c N \frac{\mathrm{e}^{\left(a-b L_{t}(i)\right)}}{1+\mathrm{e}^{\left(a-b L_{t}(i)\right)}}
$$

$c$ being the biomass growth rate, $N$ stands for total soil nitrogen available for growth ( $\mathrm{mg} \mathrm{kg}^{-1}$ soil), $a, b$ are constants. Biomass and litter are expressed in $\mathrm{g} \mathrm{m}^{-2}$.

Use of fire in natural tallgrass is mostly performed in spring in order to reduce weeds and fire velocity and enhance summer growth (Pyne et al., 1996). We simulate spring fires by removing only accumulated dead material as fuel (litter) before living biomass starts growing. The different steps of the deterministic model, developed on a lattice with $n \times n$ cells, are summarized as follows:

1. At each time step, every cell develops biomass and litter by following eqns (1) and (2).

2. Every $\theta$ iterations, burning takes place. We start by burning the middle row of the lattice. Then,

$$
L_{t}(i)=\left\{\begin{array}{cc}
L_{t}(i)(1-f) & L_{t}(i) \geqslant L_{b u r n} \\
L_{t}(i) & \text { otherwise }
\end{array}\right.
$$

where $L_{\text {burn }}$ is a constant defining some minimum litter accumulation over which a cell in position $i$ is susceptible to burn, and $f \in[0,1]$ depicts some flammability index that is inversely related to fuel moisture. That is, a cell is burned only if there is a minimum fuel load and only a fraction $f$ of this fuel will be consumed by fire. Thus, the flammability parameter depends on soil moisture or weather conditions. A flammability index has 
been formerly used in ODE models of succession for Australian forests (Possingham et al., 1995).

3. Next, fire spreads. Only those eight nextnearest-neighbor cells satisfying eq (5) will also be burned. This step is repeated until fire can no longer spread.

4. In the end, eqns (1) and (2) are computed for biomass and litter growth in every cell at this time step.

Finally, we have also introduced stochastic fluctuations on the growth rate of vegetation. This is based on one of the main features of tallgrass prairies: their high variability in primary productivity patterns (Knapp \& Smith, 2001). These authors have found values of $C V \approx 0.2$ for average annual primary production and precipitation in tallgrass prairies. We have also introduced Gaussian noise with $C V=0.2$. This random error is applied by making $c$ a Gaussian random variable $c(i)$.

To compute biomass-diversity relationships, we assumed the existence of strong postitive correlations between patch heterogeneity and species richness, as has been previously reported in tallgrass prairies (Collins, 1992). We have considered the existence of gaps when $B_{t}(i)<$ $50 \mathrm{gm}^{-2}$. Although this choice may look arbitrary, it is intended to represent some maximum light availability index below which non-dominant species may appear. In any case, it will not modify the qualitative results, in terms of spatiotemporal correlations and biomass-diversity relationships. We defined gap area entropy as a surrogate for species-richness, since gap area (i.e. number of neighboring cells pertaining to some gap) would account for some species-specific habitat availability. Significant positive spatial heterogeneity-species richness relationships have been formerly found in the Konza prairie by Collins (1992). Gap area entropy $H_{t}$ at any time was defined with the classical Shannon's index of diversity as

$$
H_{t}=-\sum_{k=1}^{A_{t}^{\max }} p_{k} \ln \left(p_{k}\right),
$$

where $A_{t}^{\max }$ is the maximum gap area represented at time $t$ and $p_{k}$ is the fraction of cells defining a gap area $k$.

\section{Results}

In our model, the continuous nature of the variables enhances the richness of spatiotemporal dynamic behaviors. Features such as percolation thresholds and complex structures may appear in a variety of states in the parametric space. For example, in high-N soils when the individual cells are mainly governed by chaotic motion in biomass and litter production, the proportion of burned patches depending on $L_{\text {burn }}$ is much affected by the period of fire $\theta$ and flammability $f$ (Fig. 1). Some of the properties that arise from applying prescribed burning in the deterministic system are shared with the pure CA approach, like the appearance of phase transitions, $L_{\text {burn }}$ being the control parameter. Furthermore, the time for fire extinction is clearly maximized when the fraction of burned sites approach $P_{\text {burned }}=0.40725$, the percolation threshold for CA models with Moore neighborhoods (i.e. those with eight adjacent cells) (Stauffer, 1985). At this point we could define some critical value of $L_{\text {burn }}$, the percolation threshold $\left(L_{c}\right)$ over which typical properties of percolation maps should appear, such as the formation of complex spatial structures between the ordered patterns governing spatial dependence and the random maps of increasing spatial heterogeneity. The pass of fire is not ensured over all cells susceptible to burn [those where $\left.L(i) \geqslant L_{\text {burn }}\right]$. At high values of $L_{\text {burn }}$, there is a high decay in the number of burned cells with respect to the number of susceptibles, which match with high fire lifetime values. Interestingly, the percolation threshold appears at these $L_{\text {burn }}$ values, because the burned structures correlate through time, so the number of actually burned cells diverges from the number of susceptibles under no-burning schemes. The percolation threshold $L_{c}$ appears when the probability of finding at least one susceptible neighbor in the no-burning scheme $P L_{t}(j) \leqslant$ $L_{\text {burn }} \leqslant, 1 / V, V=8$ being the number of neighbors in position $j$ for a Moore neighborhood.

The most important effect of prescribed burning, however, is the introduction of large correlations when increasing flammability. High $f$ values thus promote control by stabilizing the originally scattered distribution of burned cells 

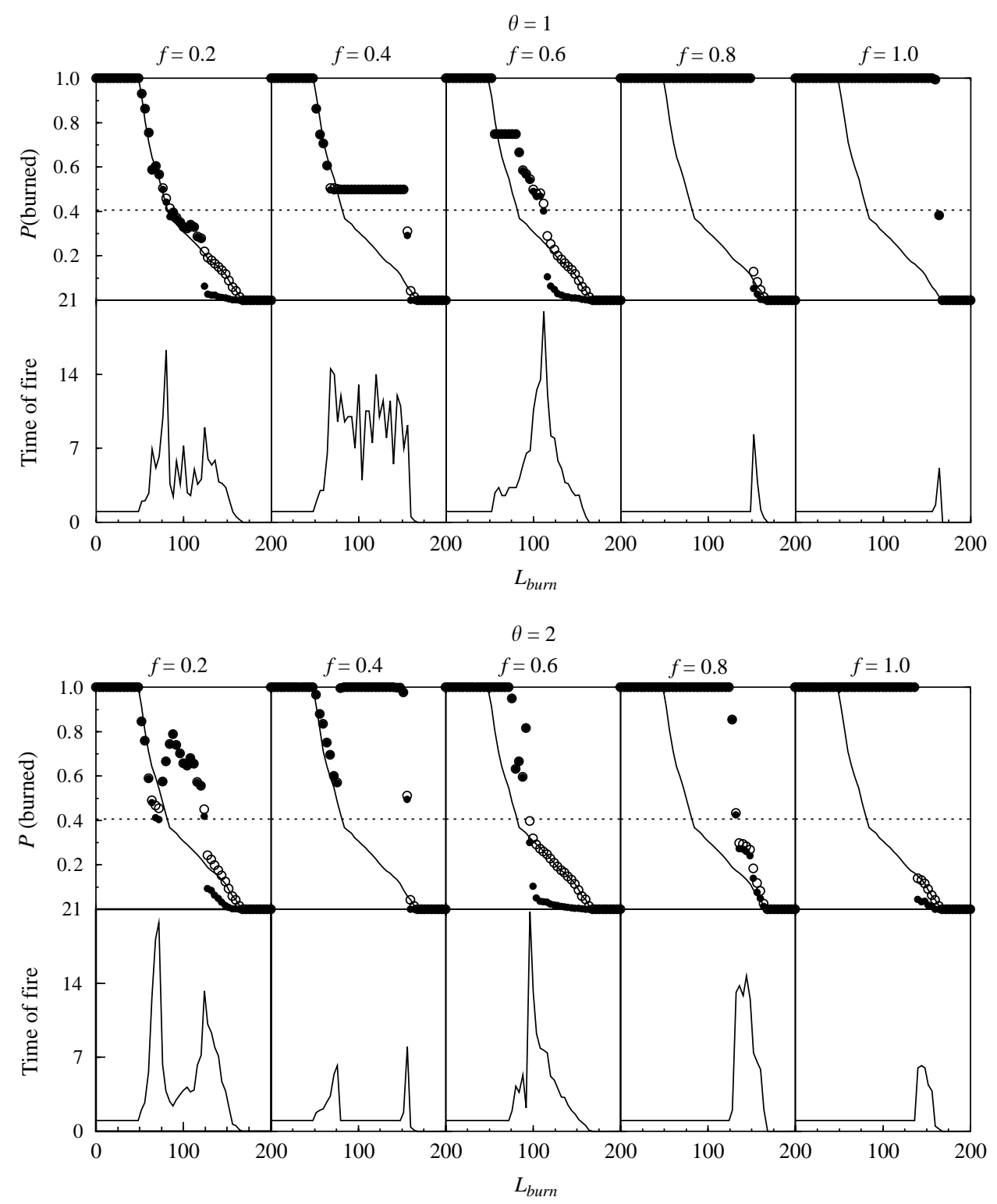

FIG. 1. Effects on a $200 \times 200$ lattice of periodic proportional disturbances by means of litter removal by fire on the high $\mathrm{N}$ scenario under burning periods $\theta=1,2$ and flammability values $f$. The rest of the parameters are the same as in

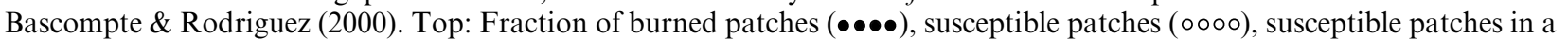
non-spatial model (i.e. no burning exerted, when $f=0)(-)$, and the percolation threshold corresponding to a Moore neighborhood (-...-). Bottom: Time to fire extinction at every $f$ recorded.

resulting from a non-controlled scheme where flammability is so low that litter is not susceptible to burn. This fact introduces high correlations among neighboring cells that trigger percolation at values of minimum litter $L_{\text {burn }}$ that were initially too low to enhance full expansion of fire. For some parameter values (i.e. $\theta=1, f=0.4$ ), burned and unburned patches may coexist in a heterogenous system for a wide range of $L_{\text {burn }}$ values, which can be seen due to the high fire lifetimes that result. Another special feature of this system is that two or more peaks in fire lifetimes and consequently, several critical values $L_{c}$ may result for burning periods $\theta>1$.

Patterns of average biomass production per cell match those of burning. Biomass is maximized at high fire frequencies and chaotic (high 
$\mathrm{N}$ availability) regimes (Fig. 2). At percolation thresholds, there are strong transitions where average biomass production declines rapidly from a controlled, correlated scheme where fire expands through the whole lattice and biomass gets maximum values to some scheme where independence of cells dominates and fire cannot spread.

Spatial patterning of vegetation (measured as large-scale spatial dependence and heterogeneity in burning patterns, and hence, in biomass production) also reflects the availability of percolation points under different burning periods and $\mathrm{N}$ availability. In a simple case assuming $f=1$, the range of $L_{b u r n}$ values over which $L_{c}$ exists is very small for short burning periods, even in the case of chaotic motion. Thus, the deterministic approach for those points $L_{\text {burn }} \approx L_{c}$ shows patterns either with full spatial correlation (i.e. where fire extends all over the landscape) or with total cell independence (where fire cannot spread) (Fig. 3). In the chaotic domain, however, a soft transition from fully burned to non-burned landscapes appears at intermediate values of $\theta$. This situation enhances the onset of percolating maps at a wide range of $L_{\text {burn }}$ values, which triggers the formation of large spatial structures and global correlations from purely internal rules.

In the stochastic counterpart, when Gaussian noise is added to local growth rates, the global appearance of biomass and burning patterns changes strongly. Stochastic fluctuations break spatial dependence in all cases, creating smallscale heterogeneity. The final maps resulting from this procedure are those where percolation arises via small, fine-grained structures. These structures are generated because random negative deviations lower some of the cell values that are above $L_{\text {burn }}$, so they no longer belong to the group of susceptibles. Thus, random noise breaks the fully burned landscape produced in the deterministic counterpart. This pattern is obvious mainly in low $\mathrm{N}$ soils and high $\mathrm{N}$ soils with low burning periods (Fig. 3). However, in high $\mathrm{N}$ soils under intermediate burning periods large-scale, not fully burned structures may develop in the deterministic case. When noise is added, these structures are broken so that smallscale heterogeneity (produced by noise) and global correlations (developed deterministically) may coexist. Interestingly, intermediate burning periods $(\theta=5,6)$ have been previously documented to maximize biomass and diversity
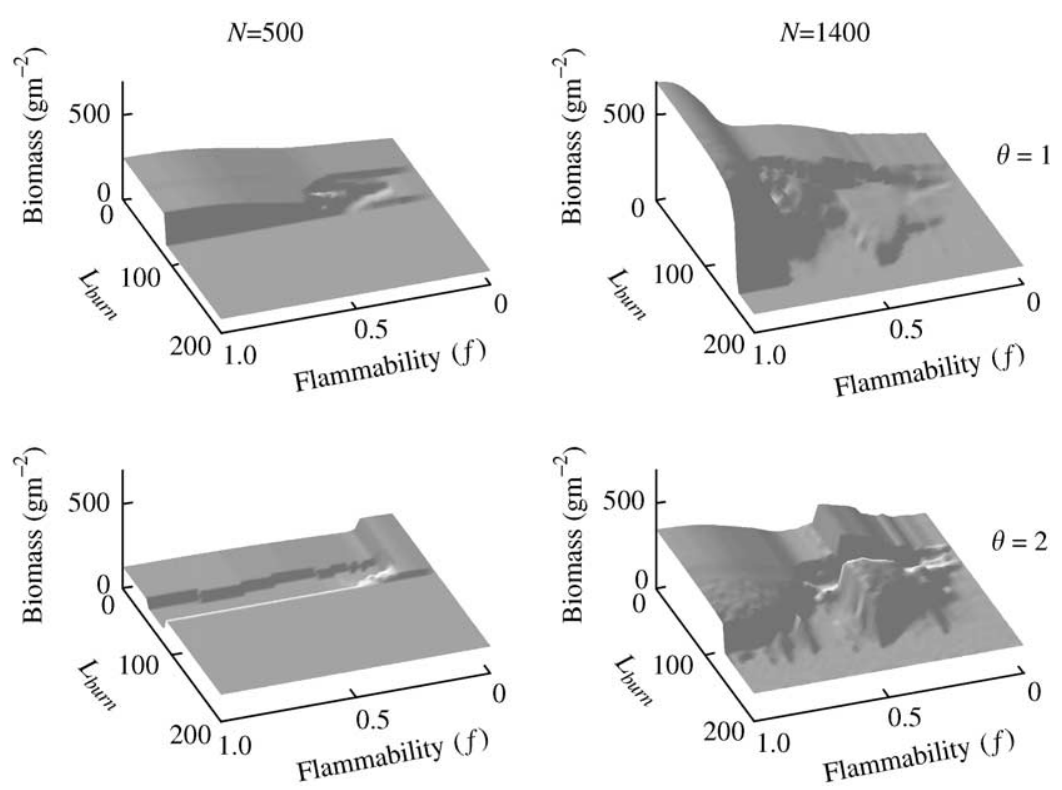

FIG. 2. Average biomass production under periodic proportional disturbances by means of litter removal by fire on the low and high $\mathrm{N}$ scenarios under burning periods $\theta=1,2$. 


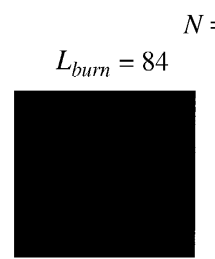

$C V=0$

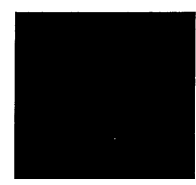

$L_{\text {burn }}=73$
$N=500$

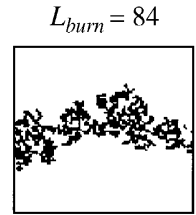

$C V=0.2$

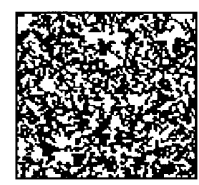

$L_{\text {burn }}=73$

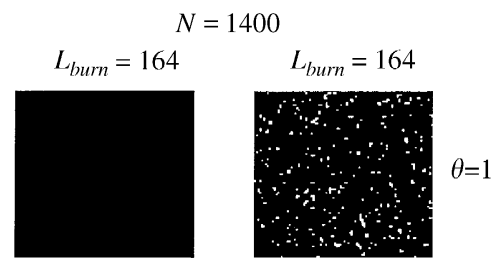

$C V=0.2$

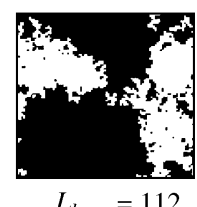

$L_{\text {burn }}=112$

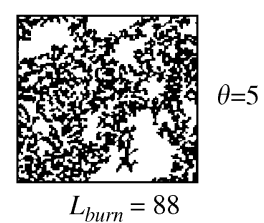

FIG. 3. Snapshots corresponding to burned areas (black cells) under different soil N, burning periods, and local stochasticity scenarios. Lattices of $100 \times 100$ cells were computed after 1200 iterations were discarded. $f=1$ in all cases.
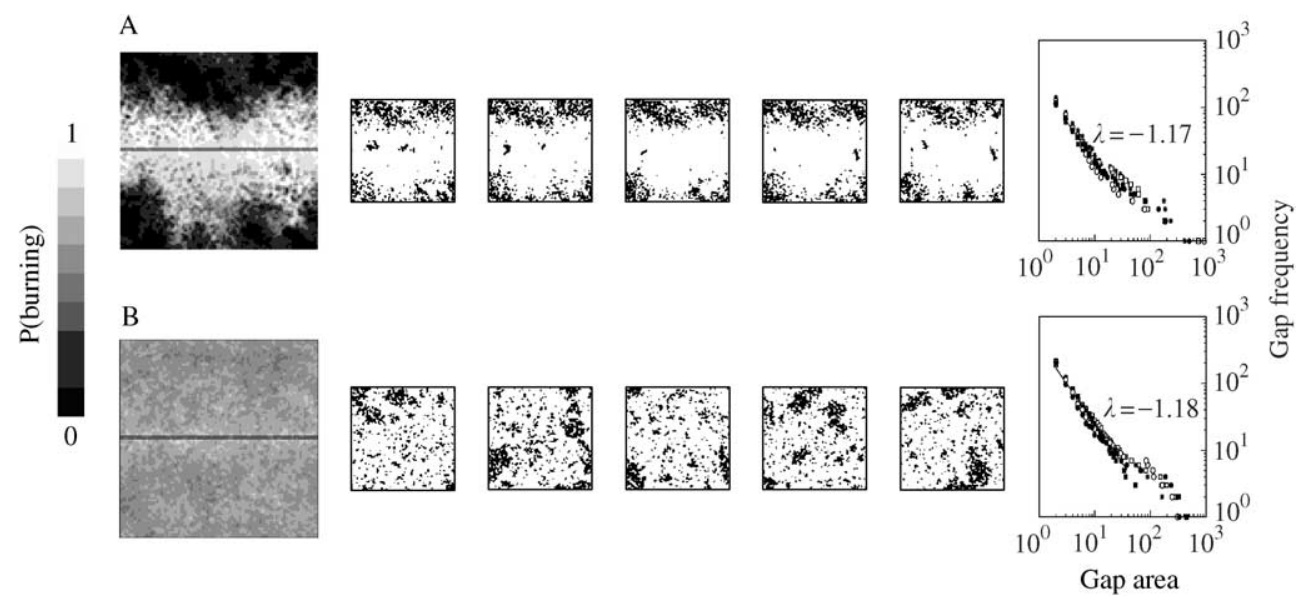

FIG. 4. Probability of burning and five consecutive snapshots in gap formation (see text) corresponding to high $\mathrm{N}$ soil conditions under burning periods $\theta=5$ years. (A) $C V=0, L_{\text {burn }}=112$. (B) $C V=0.2, L_{\text {burn }}=88$. In both cases, $f=1$. Right: gap area power law distributions. Observe that the exponent in both cases is similar.

patterns in tallgrass prairies and maintain their ecological integrity (Gibson \& Hulbert, 1987; Pyne et al., 1996).

In order to analyse spatio-temporal dependence in the dynamics of the simulated prairies, we took $P_{\text {burning }}(i)$ as the fraction of burning years along some simulation run where fire burned a specific cell at location $i$. Then we mapped the corresponding final lattices for this fraction (Fig. 4). Deterministic runs for all $\theta$ values show fully burned landscapes or strips around the initial fireline, defining large correlations through time. However, stochastic runs show, for low burning periods and low-N soils "sand-like" patterns reflecting small-scale heterogeneity and no temporal correlations in fire spread. When high- $\mathrm{N}$ soils are treated under intermediate periods of fire, large structures of cells with similar values develop, describing the formation of spatial structures, which are unstable through time, giving rise to changing schemes in the spatial spread of every fire.

Moreover, if biomass gaps (sensu Bascompte \& Rodriguez, 2000) are considered as available habitat for other species, such as forbs, we can also observe spatio-temporal correlations in diversity. While gaps can only be present under chaotic regimes (Bascompte \& Rodriguez, 2000) we found that, in fire-disturbed prairies, there are strong temporal correlations in gap formation in those landscapes frequently burned, which disappear at higher burning periods under 
local stochasticity (Fig. 4), allowing the formation of dynamic structures in habitat available for other species (Glenn \& Collins, 1990). In addition, those burning regimes that remain close to some percolation threshold reveal power-law distributions $P(A)=\sum_{A}^{A^{\prime}>A} A^{-\lambda}$ in gap area $A$ with different exponents $\lambda$. However, only the deterministic skeleton at chaotic, intermediate $\theta$ values is able to generate a wide range of percolating, power-law distributed landscapes with an exponent $\lambda \approx 1.17$ similar to that found for the model with added local Gaussian noise (Fig. 4). Fractal patterns in grassland community structure have been formerly observed (Milne, 1988; Palmer, 1992; Collins \& Glenn, 1990).

These distributions can be related to the gap area entropy and the biomass-diversity relationship. By assuming that landscape complexity enhances the specificity of niches associated to certain gap areas, we may relate plant diversity to gap area entropy. Thus, we may define biomass-gap area diversity relationships, which are strongly affected by the period of disturbance $\theta$, and consequently, by the correlations that appear at different spatial scales. We have performed simulations over a wide range of values $L_{\text {burn }} \in[0,200]$. In the deterministic case, low-N soils are characterized by low biomass and low gap area diversities. In contrast, rich-N soils only achieve a wide spectrum of gap areas at intermediate periods of disturbance (Fig. 5). This is due to the existence of a wide range of $L_{\text {burn }}$ values under which the system maintains percolating structures. When local stochasticity is at play, gap area diversity may be maximized at the percolation threshold $L_{c}$. This occurs for both $\mathrm{N}$ richness and burning

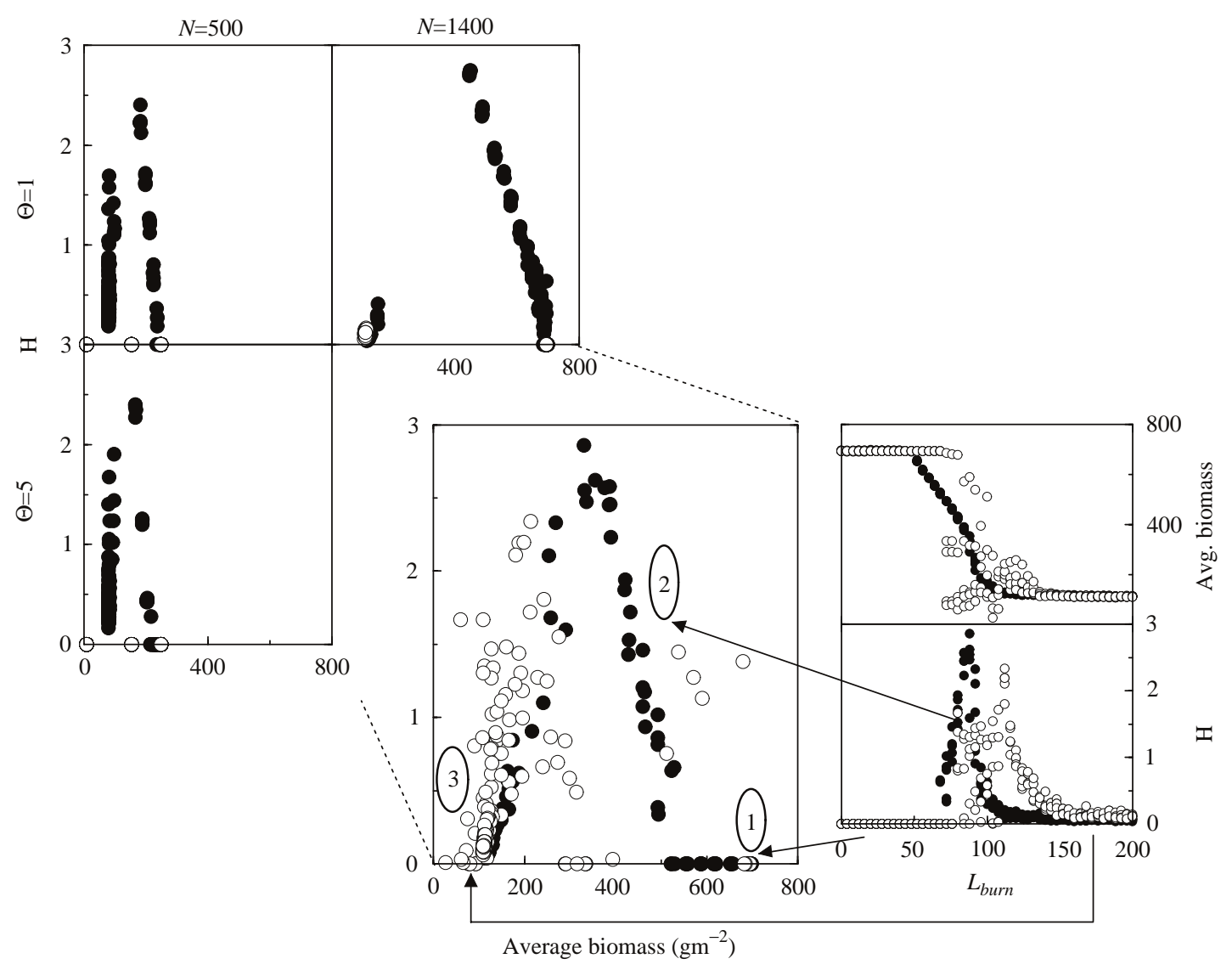

FIG. 5. Biomass-gap area entropy relationships for four scenarios with $\theta=1,5$ and $N=500,1400$ : (o००o) deterministic model $(C V=0) ;(\bullet \bullet \bullet)$ with local stochasticity $(C V=0.2)$. Observe that entropy is maximized at the percolation threshold. The numbers define different spatial behaviors: (1) Global spatial coherence; (2) coexistence of small-scale heterogeneity and spatial coherence; (3) spatial independence. 
periods. Then, maximization of diversity through gap entropy always occurs at intermediate average biomass values, which gives support to the the unimodality in biomassdiversity relationships.

However, this unimodal pattern is reflected as a direct consequence of spatial correlations arising from the burning regime. Three different regions in the biomass-diversity relationship can be observed (Fig. 5). The first region is the one described by high biomass-low diversity patterns, and arise when there is an overall synchrony in the system due to the spread of fire across the lattice. The second one, around the point of maximum diversity, corresponds to some burning regime around the percolation threshold, and may be identified by power-laws in gap area distribution, maximum fire lifetimes, and intermediate values of average biomass. The third one identifies the area where fires are not able to spread, and then all the cells behave independently, creating small-scale heterogeneities in the system, like the ones described formerly by Bascompte \& Rodriguez (2000) in their self-disturbance hypothesis. Thus, we have been able to locate every pattern of biomass and diversity as a function of the spatial correlations and, therefore, the intensity and period of disturbance applied.

\section{Discussion}

The main interactions driving the dynamics in tallgrass prairies lie on the inhibitory effect of litter accumulation on biomass production, which greatly inhibits the arrival of light and mineral nutrients to the ground, thus avoiding seed germination (Knapp \& Seastedt, 1986), which has been identified as a form of "selfdisturbance" (Bascompte \& Rodriguez, 2000) able to generate spatial heterogeneity and thus, greater diversity levels, consistent with the appearance of competition-driven core and non-equilibrium-driven satellite species (Collins \& Glenn, 1991; Glenn \& Collins, 1992).

Prescribed fires cannot be considered an experiment per se, since most of them do not face full control (i.e. stabilization of chaotic motion in all cells) on the behavior of fire and consequently, neither in biomass and litter per cell. Thus, they have to deal with local heterogeneity, but at the same time, they introduce correlations as an external, global disturbance. The spatial model of Bascompte \& Rodriguez (2000) follows a coupled-map lattice procedure, where litter dispersal is the process governing spatial patterning. We believe that some external processes such as fire, grazing or moisture conditions, should account instead for the existence of large spatial correlations, and our model explicitly includes the effect of fire for that reason. In this sense, the Bascompte and Rodriguez results, where only small-scale heterogeneities appear, are a particular set of the different possibilities available in our model.

Although the productivity-diversity relationship has been widely discussed on experimental and field grounds, some theoretical studies have also predicted unimodal and monotonic reponses in productivity-diversity (Tilman \& Pacala, 1993; Abrams, 1995; Loreau, 2000). The basis for this disagreement was taken from the different assumptions taking place, such as sampling effects or niche differentiation (Loreau, 2000). Our study focuses on populations subject to high temporal variability, and the effects that environmental disturbances at differential spatial scales, such as prescribed fires, have on the productivity-diversity relationship. This subject has been previously aimed in very few studies: Palmer (1992) found that increasing spatial dependence may generate unimodal responses in species richness.

In this paper, we have shown how different degrees of spatio-temporal dependence, driven by a gradient on fuel load and moisture conditions, introduce the full range of biomassdiversity behaviors. Three different regimes can be observed. First, one where spatio-temporal independence dominates, with a monotonic increasing response of diversity to biomass or productivity. Second, where biomass maximizes and negatively correlates with diversity, appearing at high degrees of spatio-temporal dependence. Third, where a full set of different gap areas can be observed, maximizing species diversity under the assumption of complementarity in resource area use, very close to percolation thresholds, where long spatial and temporal correlations take place. Even if chaotic 
motion, as some authors state, has not been definitely proved to appear in tallgrass prairies, the unimodal biomass-diversity pattern can be seen, even under stable motion (Fig. 5). Interestingly, our results support those of Guo \& Berry (1998) where they dissected a unimodal biomass-diversity curve in terms of spatial dependencies, in a conciliation of approaches where a general framework of conditions could provide an explanation for the observations of both unimodal and monotonic responses. Gross et al. (2000) also observed a unimodal relationship in the Konza tallgrass prairie, again suggesting strong scale dependence. A recent work on woodland colonization in tallgrass prairies has also described unimodal relationships between the fraction of habitat available and patch size heterogeneity, which interestingly maximizes at some percolation threshold (Bascompte \& Rodriguez, 2001).

Furthermore, our study points out the importance of the disturbance heterogeneity hypothesis (DHH) (Kolasa \& Rollo, 1991) as a possible mechanism for shaping spatially correlated landscapes. This model describes a quadratic relationship between the size of disturbance relative to the total area and the community heterogeneity. According to Collins (1992), our results show this relationship for all $\mathrm{N}$ treatments, though the full unimodal response seems to appear at intermediate frequencies of disturbance. Thus, there is also a frequencydependent effect of fire. Low-frequency external disturbances may maintain spatially independent landscapes under which self-disturbances are created through strong nonlinear negative feedbacks, giving rise to small-scale heterogeneities (Bascompte \& Rodriguez, 2000). In contrast, very frequent, large disturbances, spread across the landscape, and may generate unimodal responses in species richness. Here we show how spatio-temporal dependence, driven by a gradient on fuel load and moisture conditions biomass production in "monospecific" landscapes. Only at intermediate levels of disturbances (in our case, prescribed fire), diversity is maximized at intermediate values of biomass. In this case, small-scale, randomly distributed heterogeneities (generated via either deterministic nonlinearities such as the inhibitory effect of fuel accumulation or local stochasticity) break down the coalesced patches generated by fast spread of fire (Green, 1989), creating a wide mosaic of structures with different areas, where core (dominant) and satellite (rare) species could coexist. Thus, rare or non-dominant species would colonize gaps creating clumped distributions, already observed in other systems, such as tropical rainforests (Plotkin et al., 2000). In these systems, internal dynamics through mechanisms of gap-formation via tree-avalanches has been reported to produce power-law distributions of gap area (Solé \& Manrubia, 1995).

However, several studies on tallgrass prairies report burning periods of 5-6 years as the ones maximizing diversity (Gibson \& Hulbert, 1987; Pyne et al., 1996) while annual burnings (which in our model tend to create total spatial dependence and maximum biomass under high fuel flammability) maximize ANPP (Briggs \& Knapp, 1995). Others have found that spatial dependence and heterogeneity are maximized just after a fire event (Henebry, 1995). Thus, external environmental variability, which seems to be extremely important in shaping tallgrass landscapes (Knapp \& Smith, 2001) mixed with local stochasticity could be the key factors determining fire-spread avalanches in many grasslands. Whether fire is an intrinsic property of these systems is the question that should be answered in order to infer some self-organized criticality (SOC) properties governing the dynamics of biomass and diversity. The extremely irregular nature of drought events in prairies may widen the range of among-year flammability variability. Then, under a wide set of conditions (i.e. low flammability and $\theta=2$ ), the possibility of percolating landscapes increases, so fractality in gap formation would appear as a general and plausible consequence of fire regimes.

Another important source of broad-scale disturbances that has been addressed in tallgrass prairies is grazing (Knapp et al., 1999). They showed that bison is attracted by recently burned sites and that it selectively removes grasses from this areas. Under this assumption, the grazing-fire interaction could generate much more heterogeneity in burned areas where spatial correlations are generated. However, grazing 
patches are reselected from year to year by $50 \%$, so grazing tends to also correlate with the areas that are disturbed. Further theoretical studies are needed for a general understanding of the grazing-fire interaction in grassland ecosystems and how this relation affects productivitydiversity responses. Our work emphasizes the role of nonlinearities and phase transitions governing ecological processes and the scale of observation within the landscape. Although fire and grazing disturbances are different processes affecting species diversity, both are broad-scale agents coalescing landscapes defined by smallscale individual units. Thus, both may constrain smaller-scale patterns (Collins \& Glenn, 1990). Our results prompt the need for further experiments at the landscape level to check the role of the effect of large, correlating disturbances on the biomass-diversity response. Former experiments dealing with small, independent plots might be masking this response through the socalled sampling effect. Many of them have been designed on independent plots in order to avoid pseudoreplication. In such a case, monotonic biomass-diversity relationships such as the ones reported in the literature would be a logical consequence arising from this spatial independence. Thus, the use of multiscale analyses is further needed in experimental protocols, and further studies should account for scale dependence by studying diversity at multiple scales (Milne, 1992).

The authors are greatly indebted to the Santa $\mathrm{Fe}$ Institute for partial support during our stay at New Mexico. M. A. Rodriguez, J. Bascompte, B. Milne, J. M. Montoya and S. Naeem greatly improved the initial work. Partial computing facilities were due to the grant DGYCIT PB97-0693.

\section{REFERENCES}

Abrams, P. A. (1995). Monotonic or unimodal diversityproductivity gradient: what does competition theory predict? Ecology 76, 2019-2027.

Axelrod, D. I. (1985). Rise of the grassland biome, central North America. Bot. Rev. 51, 163-201.

BAK, P., Chen, K. \& TANG, C. (1990). A forest-fire model and some thoughts on turbulence. Phys. Lett. A 147, 297-300.

BAscompte, J. \& Rodriguez, M. A. (2000). Selfdisturbance as a source of spatiotemporal heterogeneity: the case of the tallgrass prairie. J. theor. Biol. 204, 153-164, doi:10.1006/jtbi.2000.2002.

Bascompte, J. \& Rodriguez, M. A. (2001). Habitat patchiness and plant species richness. Ecol. Lett. (in press).

BrigGs, J. M. \& KNAPP, A. K. (1995). Interannual variability in primary production in tallgrass prairie: climate, soil moisture, topographic position and fire as determinants of aboveground biomass. Am. J. Bot. 82, 1024-1030.

Collins, S. L. (1992). Fire frequency and community heterogeneity in tallgrass prairie vegetation. Ecology $\mathbf{7 3}$, 2001-2006.

Collins, S. L. (2000). Disturbance frequency and community stability in native tallgrass prairie. Am. Nat. 155, 311-325.

Collins, S. L. \& Glenn, S. M. (1990). A hierarchical analysis of species' abundance patterns in grassland vegetation. Am. Nat. 135, 633-648.

Collins, S. L. \& Glenn, S. M. (1991). Importance of spatial and temporal dynamics in species regional abundance and distribution. Ecology 72, 654-664.

Culver, D. (1998). Literature review of fire ecology and effects. United States Air Force Academy. Colorado Springs. Colorado. The Colorado Natural Heritage Program Research Report No. 2. Fort Collins, CO.

Daubenmire, R. (1968). Ecology of fire in grasslands. In: Advances in Ecological Research, Vol. 5, (Cragg, J.B., ed.), pp. 209-266, New York: Academic Press.

Desharnais, R. A., Constantino, R. F., Cushing, J. M., Henson, S. M. \& Dennis, B. (2001). Chaos and population control of insect outbreaks. Ecol. Lett. 4, 229-235.

Drossel, B. \& Schwabl, F. (1992). Self-organized critical forest-fire model. Phys. Rev. Lett. 69, 1629-1632.

Gamarra, J. G. P., Solé, R. V. \& Alonso, D. (2001). Control, synchrony and the persistence of chaotic populations. Chaos Solitons Fractals 12, 235-249.

Gibson, D. J. \& Hulbert, L. C. (1987). Effects of fire, topography and year-to-year climatic variation on species composition in tallgrass prairie. Vegetatio $\mathbf{7 2}$, 175-185.

Glenn, S. M. \& Collins, S. L. (1990). Patch structure in tallgrass prairies: dynamics of satellite species. Oikos $\mathbf{5 7}$, 229-236.

Glenn, S. M. \& Collins, S. L. (1992). Effects of scale and disturbance on rates of immigration and extinction of species in prairies. Oikos 63, 273-280.

Green, D. G. (1989). Simulated effect of fire, dispersal and spatial pattern on competition within vegetation mosaics. Vegetatio 82, 139-153.

GreEN, D. G. (1990). Landscapes, cataclysms and population explosions. Math. Comput. Model. 13, 75-82.

Gross, K. L., Willig, M. R., Gough, L., Inouye, R. \& Cox, S. B. (2000). Species diversity and productivity at different spatial scales in herbaceous plant communities. Oikos 89, 417-427.

GuO, Q. \& BERrY, W. L. (1998). Species richness and biomass: dissection of the hump-shaped relationships. Ecology 79, 2555-2559.

Hector, A. et al. (1999). Plant diversity and productivity of European grasslands. Science (Wash) 286, 1123-1127.

Henebry, G. M. (1995). Spatial model error analysis using autocorrelation indices. Ecol. Model. 82, 75-91. 
Hurtt, G. C. \& Pacala, S. W. (1995), The consequences of recruitment limitation: reconciling chance, history, and competitive differences between plants. J. theor. Biol. 176, 1-12, doi:10.1006/jtbi.1995.0170.

Huston, M. A. (1994). Biological Diversity. Cambridge, U.K.: Cambridge University Press.

Johnson, K. G., Vogt, K. A., Clark, H. J., Schmitz, O. J. \& VoGT, D. J. (1996). Biodiversity and the productivity and stability of ecosystems. Trends Ecol. Evol. 11, 372-377.

Knapp, A. K., Blair, J. M., Briggs, J. M., Collins, S. L., Hartnett, D. C., Johnson, L. C. \& Towne, E. G. (1999). The keystone role of bison in North American tallgrass prairie. BioScience 49, 39-50.

KnapP, A. K. \& Seastedt, T. R. (1996). Detritus accumulation limits productivity of tallgrass prairie. BioScience 36, 662-668.

Knapp, A. K. \& Smith, M. D. (2001). Variation among biomes in temporal dynamics of aboveground primary production. Science (Wash) 291, 481-484.

Kolasa, J. \& Rollo, C. D. (1991). Introduction: the heterogeneity of heterogeneity: a glossary. In: Ecological Heterogeneity (Kolasa, J. \& Pickett, S.T.A., eds), pp. 1-23. New York, NY, U.S.A.: Springer-Verlag.

Loreau, M. (2000). Biodiversity and ecosystem functioning: recent theoretical advances. Oikos 91, 3-17.

Malamud, B. D., Morein, G. \& Turcotte, D. L. (1998). Forest fires: an example of self-organized critical behaviour. Science (Wash) 281, 1840-1842.

Milchunas, D. G., Sala, O. E. \& Lauenroth, W. K. (1988). A generalized model of the effects of grazing by large herbivores on grassland community structure. Am. Nat. 132, 87-106.

Milne, B. T. (1988). Measuring the fractal geometry of landscapes. Appl. Math. Comput. 27, 67-79.

Milne, B. T. (1992). Spatial aggregation and neutral models in fractal landscapes. Am. Nat. 139, 32-57.

Naeem, S., Thompson, L. J., Lawler, S. P., Lawton, J. H. \& Woodfin, R. M. (1994). Declining biodiversity can alter the performance of ecosystems. Nature $\mathbf{3 6 8}$, 734-737.

O’Lear, H. A., Seastedt, T. R., Briggs, J., Blair, J. M. \& Ramundo, R. A. (1996). Fire and topographic effects on decomposition rates and nitrogen dynamics of buried wood in tallgrass prairie. Soil Biol. Biochem. 28, 323-330.

Palmer, M. W. (1992). The coexistence of species in fractal landscapes. Am. Nat. 139, 375-397.

Plotkin, J. B., Potts, M. D., Leslie, N., Manokaran, N., LaFrankie, J. \& Ashton, P. S. (2000). Species-area curves, spatial aggregation, and habitat speciation in tropical forests. J. theor. Biol. 207, 81-99, doi:10.1006/ jtbi.2000.2158.

Pyne, S. J., Andrews, P. J. \& Laven, R. D. (1996). Introduction to Wildland Fire, 2nd Edn., 769pp. New York, U.S.A.: John Wiley \& Sons.

Possingham, H. P., Comins, H. N. \& Noble, I. R. (1995). The fire and flammability niches in plant communities. J. theor. Biol. 174, 97-108, doi:10.1006/jtbi.1995.0082.

Rosenzweig, M. L. \& Abramsky, Z. (1993). How are diversity and productivity related? In: Species Diversity in Ecological Communities (Ricklefs, R.D. \& Schluter, D., eds), pp. 52-65. Chicago, IL, U.S.A.: University of Chicago Press.

Seastedt, T. R. \& Knapp, A. K. (1993). Consequences of non-equilibrium resource availability across multiple time scales: the transient maxima hypothesis. Am. Nat. 141, 621-633.

Solé, R. V. \& Alonso, D. (2000). Habitat fragmentation and biodiversity collapse under recruitment limitation. Santa Fe Institute Working Paper 00-12-066.

Solé, R. V., Gamarra, J. G. P., Ginovart, M. \& Lopez, D. (1999). Controlling chaos in ecology: from deterministic to Individual-based Models. Bull. Math. Biol. 61, 1187-1207.

SolÉ, R. V. \& Manrubia, S. C. (1995). Are rainforests selforganized in a critical state? J. theor. Biol. 173, 31-40, doi:10.1006/jtbi.1995.0040.

Stauffer, D. (1985). Introduction to Percolation Theory. London, England: Taylor \& Francis.

Tilman, D. (1982). Resource Competition and Community Structure. Princeton, NJ, U.S.A.: Princeton University Press.

Tilman, D. (1994). Competition and diversity in spatially structured habitats. Ecology 75, 2-12.

Tilman, D. \& Pacala, S. (1993). The maintance of species richness in plant communities. In: Species Diversity in Ecological Communities (Ricklefs, R.D. \& Schluter, D., eds), pp. 13-25. Chicago, IL, U.S.A.: University of Chicago Press.

Tilman, D. \& Wedin, D. (1991). Oscillations and chaos in the dynamics of a perennial grass. Nature 353, 653-655.

Tilman, D., Wedin, D. \& Knops, J. (1996). Productivity and sustainability influenced by biodiversity in grassland ecosystems. Nature 379, 718-720.

Waide, R. B., Willig, M. R., Steiner, C. F., MittelBaCh, G., Gough, L., Dodson, S. I., Juday, G. P. \& Parmenter, R. (1999). The relationship between productivity and species richness. Annu. Rev. Ecol. Syst. 30, 257-300. 